\title{
Do Simple Objects Facilitate Infants' Formation of a Spatial Category?
}

\author{
Youjeong Park \\ Marianella Casasola \\ Cornell University \\ Jinwook Kim \\ Seoul National University
}

\begin{abstract}
The present study investigated infants' ability to form a category of a support relation (i.e., "on") when the objects depicting the relation were perceptually simple versus more complex. Twenty Korean infants of 14 months were habituated to dynamic support events with objects that were either simple or more complex in appearance. They were then tested with events that differed from the habituation events in the specific objects, spatial relation, or both. Infants formed a support category whether familiarized to simple or complex objects, looking significantly longer at test events with a novel than familiar relation. The results indicate that at 14 months of age, object features do not impact infants' ability to form a categorical representation of support.
\end{abstract}

Key words : categorization, simplicity, generalization, object features, spatial cognition

Categorization is an essential cognitive ability that enables us to generalize our experiences from a set of exemplars to novel exemplars. In the domain of relations, a categorizer generalizes a relation among known objects or elements to novel ones responding to the familiarized relation as familiar despite the novelty of the elements depicting it. In the present study, we examine infants' ability to form a categorical representation of a small-scale spatial relation, namely, support (i.e., one object placed on another object). Research on infants' ability to categorize spatial relations thus far has mainly focused on what spatial categories infants form at different points in development (e.g., BehlChadha \& Eimas, 1995; Casasola, Cohen, \& Chiarello, 2003; Gava, Valenza, \& Turati, 2009;

* Corresponding Author: Youjeong Park, Department of Human Development, B52 MVR Hall, Cornell University, Ithaca, NY 14853 , USA. E-mail: yp229@cornell.edu
Quinn, 1994; Quinn, Adams, Kennedy, Shettler, \& Wasnik, 2003; for a review, see Casasola, 2008), while only a few studies have examined how infants form a spatial category (e.g., Casasola, 2005; Quinn, Cummins, Kase, Martin, \& Weissman, 1996; Quinn, Polly, Furer, Dobson, \& Narter, 2002). To fill this empirical gap and provide insights into the ideal conditions for infants' spatial learning, we test infants' ability to categorize a support relation under two learning conditions: when infants viewed the spatial relation with perceptually simple objects versus more complex objects. Our goal was to explore whether the simplicity of objects enhances infants' formation of a category of support.

Recently, researchers have begun to explore how the perceptual simplicity of learning materials influences category learning and generalization (e.g., Kaminski, Sloutsky, \& Heckler, 2006, 2008; Kaminski \& Sloutsky, 
2010; Rattermann, Gentner, \& DeLoache, 1990; Son, Smith, \& Goldstone, 2008, 2011; Uttal, Scudder, \& DeLoache, 1997; Welling, 2011). Several studies have documented an advantage of simple objects over complex ones in generalization tasks. For example, in a word learning study, Son, Smith, and Goldstone (2008) found that toddlers demonstrated more robust generalization of an object label from a single instance to new examples when the single instance (i.e., the training object) was perceptually sparse and had only the overall geometric shape of the target object. In contrast, toddlers had more difficulty generalizing the object label when the training instance was complex, with rich details. These researchers proposed that simplified objects shortcut the category learning process and foster generalization because they omit categoryirrelevant elements and depict only categoryrelevant information, thereby providing alreadyabstracted instances. In contrast, more perceptually complex objects contain categoryirrelevant details and these details seem to hinder learners' formation of an abstract categorical representation (Son, Smith, \& Goldstone, 2008).

Similar advantages of perceptually simple objects have been found in studies examining the learning of relational categories (Amaya, Uttal, O’Doherty, Liu, \& DeLoache, 2008; Kaminski \& Sloutsky, 2010; Kaminski, Sloutsky, \& Heckler, 2006, 2008; Rattermann, Gentner, \& DeLoache, 1990; Son, Smith, \& Goldstone, 2011; Welling, 2011). First, Rattermann, Gentner, and DeLoache (1990) found that, in a relational mapping task where the relation match was pitted against the object match, young children made more relational choices when the objects were perceptually sparse than when they were perceptually rich. Similarly, Kaminski and colleagues demonstrated that both adults (Kaminski, Sloutsky, \& Heckler, 2008) and children (Kaminski \& Sloutsky, 2010; Kaminski, Sloutsky, \& Heckler, 2006) generalized relational rules to new instances with greater ease when the rules were demonstrated by abstract symbols than concrete objects. Moreover, Amaya and colleagues (2008) investigated elementary school children's use of manipulatives in mathematical problem solving, and found an effect of the object features in children's ability to focus on the relevant mathematical task. Specifically, children who were given more attractive blocks that were patterned with bright colors were more likely to play with the blocks and be diverted from the relevant mathematical concepts than children who were given less exciting monochromatic blocks. Together, these researchers have argued that perceptually simple objects are more beneficial because they are less interesting in their own right as objects. Thus, they do not detract attention away from the relational information. In contrast, perceptually rich objects draw more attention to themselves and consequently may hinder relational thinking (Kaminski, Sloutsky, \& Heckler, 2006, 2008; Rattermann et al., 1990).

In sum, studies that have examined generalization of objects and relational information suggest that there are benefits to perceptually simple exemplars. One advantage is providing a ready-made abstraction through the removal of irrelevant information. The other advantage is more specific to relational categories, namely, the ability to look beyond the component objects and focus on the relation. Because simple objects, more than perceptually rich object, are less likely to overshadow the relations, they may play a critical role in promoting attention to relations and consequently generalization.

Despite findings pointing to the merits of simple objects in fostering generalization, there are studies that outline a more nuanced picture and suggest that simple objects may not always provide an advantage over complex objects (Paik \& Mix, 2006; Petersen \& McNeil, 2008; Son, Smith, \& Goldstone, 2011; Welling, 2011). For instance, Petersen and McNeil (2008) reported that the beneficial effect of simplicity occurs only when the complex objects have established meanings as objects. More specifically, they found that preschoolers' counting performance was significantly hampered by complex objects that had established meanings (e.g., animal 
replicas), but not by complex objects lacking an established meaning (e.g., sparkly, decorated pom poms). Second, Welling (2011) found a developmental difference in the effect of simplicity. Although 4-year-olds generalize dynamic relations better when the relations were depicted with generic shapes, simplicity did not matter for 3-year-olds. The younger children failed to generalize in both conditions and interestingly learned the labels for dynamic relations slightly better when the relations were depicted with concrete, perceptually rich objects. Also, Son, Smith, and Goldstone (2011) found no difference between simple and complex objects in a relation-matching task when the relation exemplars varied from trial to trial. However, when a single exemplar of a relation was used across all trials, children still performed better with a simple shape as the exemplar. Thus, there are some inconsistent findings, and these findings together suggest that there can be instances in which complex objects may be equally effective or slightly better suited for learning relations than simple objects.

Building on these previous findings, we hypothesize that similar benefits of simplicity may be found in infant spatial categorization. While a spatial event always consists of objects that comprise a spatial relation, forming a spatial category from multiple exemplars requires learners to look beyond the objects and note the spatial relation between the objects. This might be a challenging task for infants, given that even older children have difficulty ignoring the objects in relation match tasks (Rattermann, Gentner, \& DeLoache, 1990) and that infants who fail to discriminate the spatial relations successfully discriminate the objects (e.g., Casasola \& Cohen, 2002). If objects do overshadow spatial relations, then spatial events that present perceptually simple objects may facilitate infants' attention to and abstraction of the relevant information, resulting in infants more easily forming an abstract categorical representation of a spatial relation. In contrast, viewing spatial events with complex objects could require infants to direct more attention and cognitive processing of the objects, and thus hampers infants' attention to the relational commonality across the events.

There is previous evidence to support this possibility. In one study, infants of 14 months formed a support category when habituated to two exemplars of the support relation but not when habituated to six exemplars (Casasola, 2005, but see Casasola \& Park, in press). Because each exemplar presented a distinct pair of objects, the increase in the exemplars in the six-exemplar condition might have presented too much object information for infants to attend to the spatial relation and form the spatial category. A similar finding was reported in a study examining toddlers' learning of a motion category (Maguire, Hirsh-Pasek, \& Golinkoff, 2008). Since perceptual complexity versus simplicity of objects is another way to manipulate the amount of object information, it is possible that 14-month-old infants will respond similarly to the simple versus complex objects in forming a spatial category of a support relation. That is, infants may be distracted from the relational nature of the task with complex objects and may best form a support category when simple objects depict the support relation.

In the present study, we directly test this possibility, focusing on 14-month-old Koreanlearning infants' formation of a support category (i.e., a category of on). We focus on a support relation because prior research indicated that the category of support is relatively challenging for infants to form and that infants' formation of a support category can be sensitive to learning conditions (Casasola, 2005). Although infants can form an abstract category of containment as early as 6 months (Casasola \& Cohen, 2002; Casasola, Cohen, \& Chiarello, 2003), their formation of an abstract category of support remains fragile even at 18 months (Casasola \& Cohen, 2002). However, when habituated with perceptually similar objects, infants of 14 months (Casasola, 2005; Casasola \& Park, in press) and even those at10 months can form a support category in certain cases (Casasola \& Park, in press). Similarly, we chose to test infants of 14 months because at this age, infants' support categorization can be influenced by the number 
of exemplars presented during habituation (Casasola, 2005; but see Casasola \& Park, in press). Thus far, research on infant spatial categorization has been conducted mostly with English-learning infants in the US. This focus on English-learning infants may limit generalizing the results to infants in other cultures. We thus focus on infants in Korea and assess whether the present results mirror those obtained in a study with English-learning infants (Park \& Casasola, in preparation). Recently, researchers have begun to study Korean infants' categorization of spatial relations mainly for the purpose of examining the effect of language-specific input on infant categorization (e.g., Choi, 2006; McDonough, Choi, \& Mandler, 2003). We thus contribute to this body of work by including infants from Korea and can address whether any differences emerge for a support relation that is described in a semantically similar manner in English and Korean (Choi \& Bowerman, 1991). In sum, our results can address whether Korean infants benefit most from simple than complex objects in forming a category of support because simple objects lack superfluous features and may minimize infants' attention to the objects themselves and promote attention to the spatial relation between the objects.

\section{Method}

\section{Participants}

Participants were twenty Korean 14-monthold infants, 8 females and 12 males, living in Seoul (the capital) or Wonju (a middle-size city) in South Korea. Infants ranged in age from 13.3 to 14.7 months ( $M=14.1$ months, $S D=0.4$ months) and all were learning Korean as their native language. Data from an additional 6 infants were collected but excluded for the following reasons: fussiness $(n=2)$, failure to meet the habituation criterion, which is described below $(n=1)$, looking time to the familiar test event that was more than two standard deviations above the mean $(n=2)$, and parental interference $(n=1)$. Families were recruited primarily by visiting childcare centers, but also through an advertisement post on a Korean website of parents. Most families were middle class, and the majority of parents were college-educated (2 years or 4 years of college education). All infants received an infant $\mathrm{T}$-shirt or sippy cup in appreciation for participation.

\section{Stimuli}

Two sets of stimuli, one designed to be perceptually simple and another designed to be perceptually more complex, were created for the study. Each set consisted of six pairs of objects and each pair consisted of a figure and a referent object. The figures were small blocks, $6 \times 6 \times 10$ $\mathrm{cm}$, while the referents were larger, tissue boxes, $23.5 \times 10.5 \times 8.5 \mathrm{~cm}$. The opening in the tissue boxes allowed the figure to be inserted into the referent box to demonstrate a containment relation. Inverting the referent object allowed the figure object to be supported in the center of the referent object to demonstrate a support relation. In the simple set, both the figures and referents were a solid color, wrapped only in a monochromatic, nonwoven fabric without decoration. In the complex set, the figures and the referents were similar in color to the simple set but were further decorated with bright patterns (e.g., dots, wave, checkerboard, circle) and appendages (e.g., arms, wings, antennas, see Figure 1).

All events began with the figure to the right of the referent. A hand then appeared from the right, lifted the figure, and placed it in either a support (on) or containment (in) spatial relation to the referent. Each event was videotaped using a Canon ZR85 digital video camera. The events were then transferred into a Macintosh computer and converted to Quicktime movies. Each event was approximately $7 \mathrm{~s}$. For a trial, a particular event was looped five times to create a trial that was $35 \mathrm{~s}$ in duration.

\section{Apparatus}

Testing took place in a small, quiet room in the child's daycare center or home. A folding 
Do Simple Objects Facilitate Infants' Formation of a Spatial Category?

Habituation Events: Simple Condition
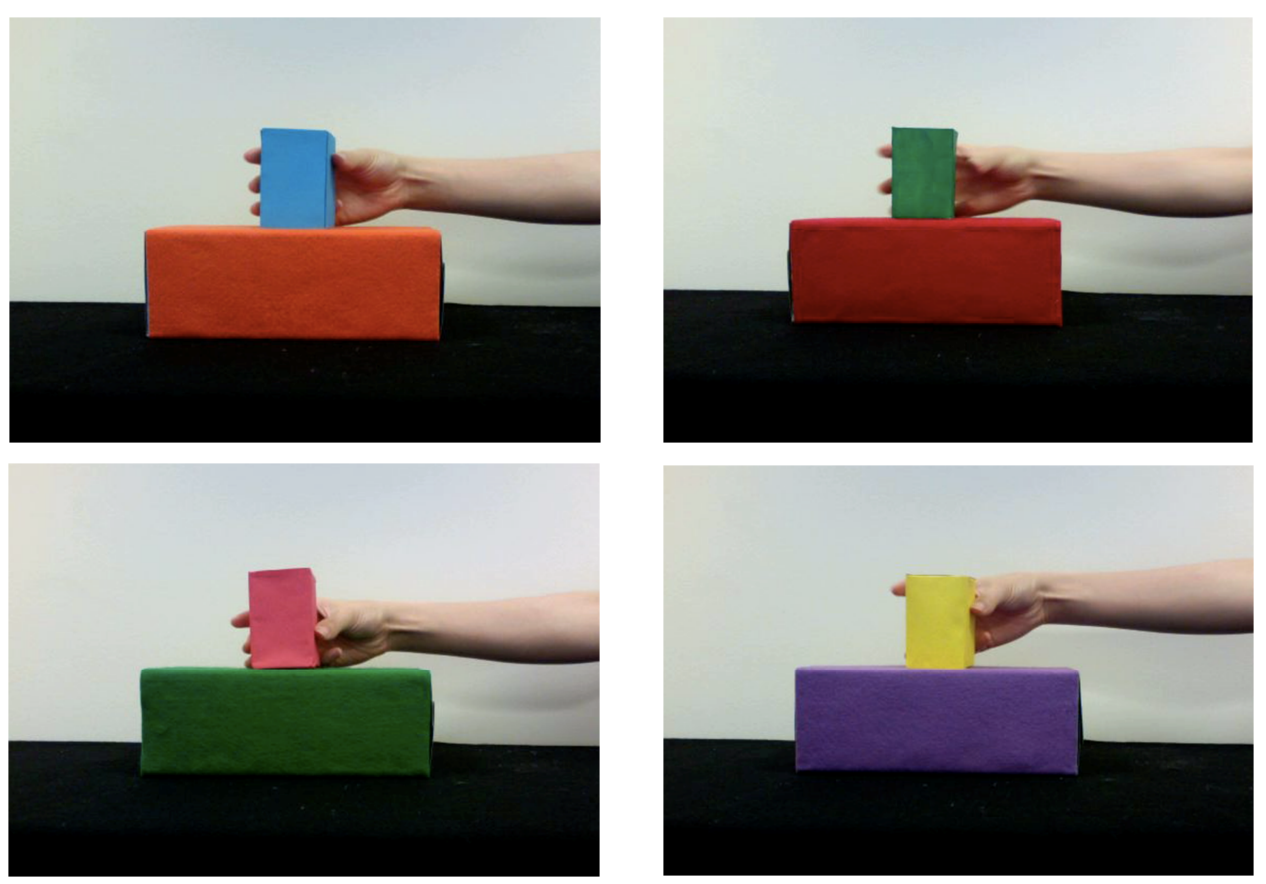

Test Events: Simple Condition
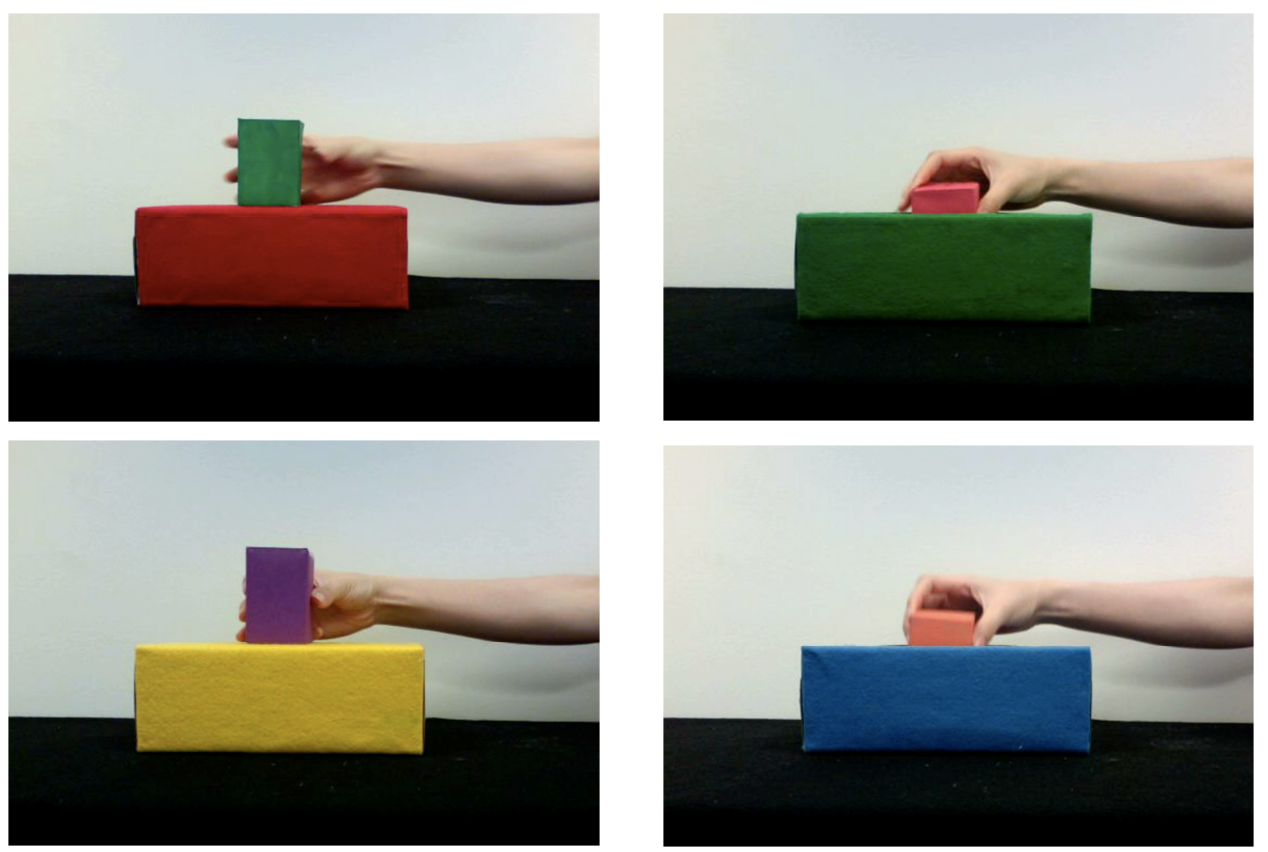

Child Studies in Diverse Contexts, 2012, 2(2) 
Park, Casasola, \& Kim

Habituation Events: Complex Condition
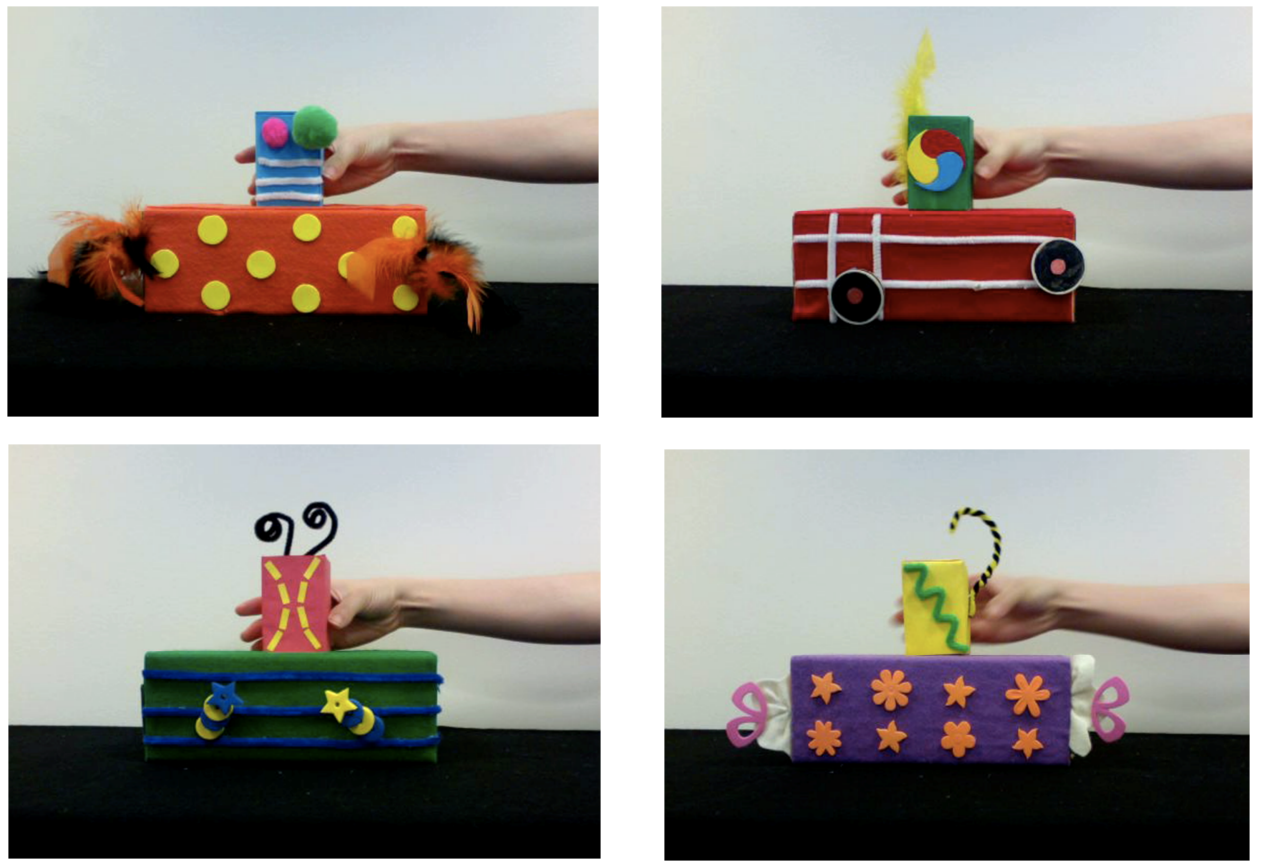

Test Events: Complex Condition
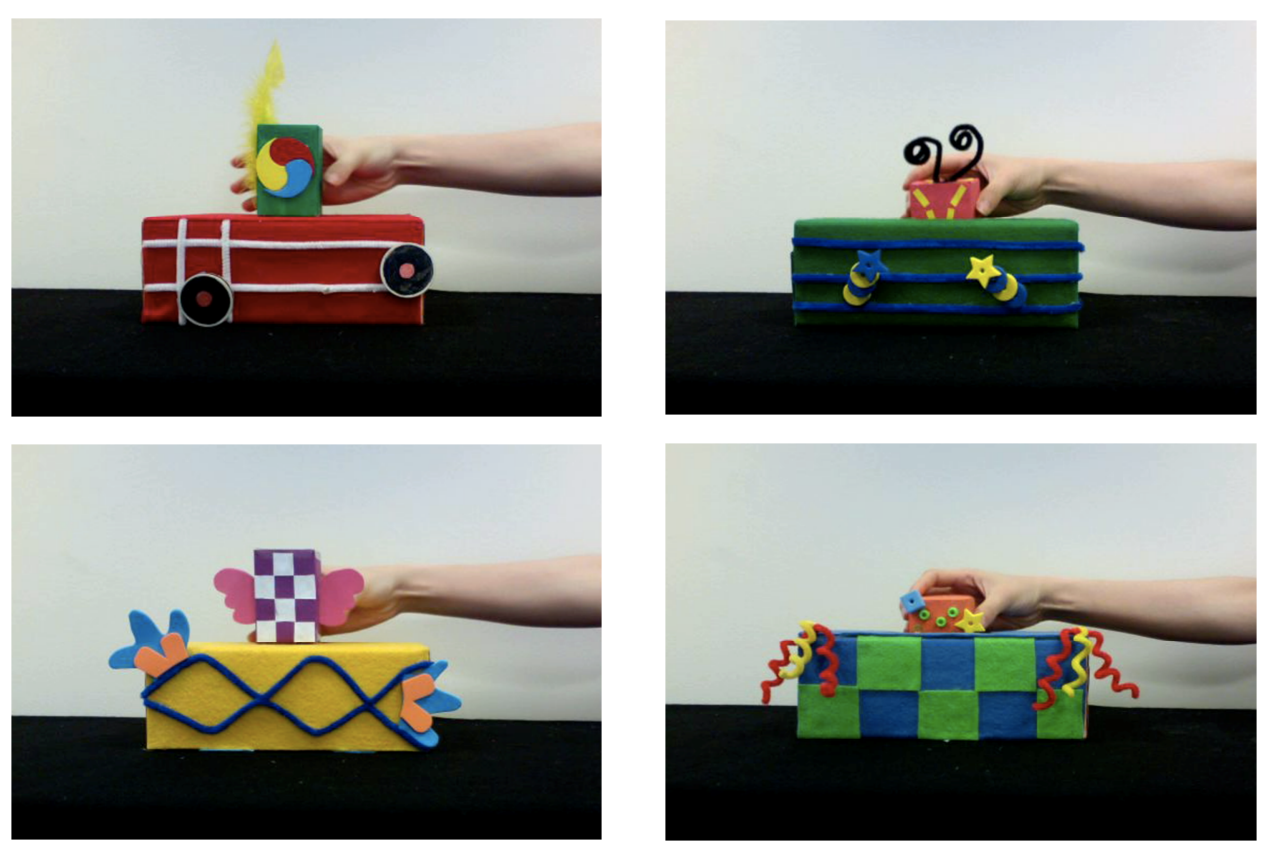

Figure 1. Sample habituation and test events presented in each condition 
table $(120 \mathrm{~cm} \times 60 \mathrm{~cm} \times 70 \mathrm{~cm})$, covered with the black tablecloth, was used to place a $50 \mathrm{~cm}$ Dell monitor, on which all events were presented. The tablecloth reached to the floor and $6 \mathrm{~cm}$ hole in the black tablecloth underneath the monitor allowed Sony video camera to focus on the infant's eye movements. The Sony video camera as linked to a Macbook computer located under the folding table, allowing the experimenter to monitor infant looking to the stimulus events. The laptop computer was also used to run the Habit software (Cohen, Atkinson, \& Chaput, 2000), a program used to present the video events on the monitor and record infant looking times during the procedure. A chair was placed $120 \mathrm{~cm}$ away from Dell monitor for the parent to sit with their infant.

\section{Procedure}

Two experimenters collaborated to run the experiment for all infants. Prior to testing, the experimenters explained the purpose and procedure of the study to a parent or a daycare teacher. Each parent provided written consent and was carefully instructed to avoid any behaviors that could influence infants' looking behavior. Then one experimenter went to the testing room and hid under the table that was covered by black cloth. After that, the other experimenter guided the infant and parent to the testing room and left the room. Each experimenter maintained the same role for all infants. During the experiment, infants sat on their parents' laps facing the monitor on which the events were presented. The experimenter hidden under the table observed infant fixations to the events via the video camera. The experimenter could view only the infant through the camera and remained blind to the specific stimulus presented.

The experimenter began the testing procedure by initiating the Habit program. Upon doing so, an attention-getter appeared on the monitor and remained on the screen until infants directed their attention to the monitor. Once infant attended to the stimulus on the monitor, the experimenter depressed one key on the notebook keyboard and a stimulus event replaced the attention-getter on the monitor. The event remained on the monitor for as long as the infant attended to it. Once infants looked away from the event for at least one consecutive second or longer, the event ended. If infants looked for the full duration of the trial, the event ended after it had played for the full $35 \mathrm{~s}$ duration of the trial.

Infants were randomly assigned to one of either the simple or complex condition. Infants in the simple condition viewed only the spatial events with simple objects during habituation while those in the complex condition viewed only the spatial events with complex objects. As the first trial of the experiment, infants viewed a pretest trial, a video of a pink pig moved by a hand in a zigzag path. Next, the habituation phase began. All infants were habituated to the four dynamic support relation events, chosen at random from the possible six support events created. Infants viewed the four habituation events in a semi-randomized order across blocks of four trials until their total looking time during three consecutive trials was less than half of total looking time during the first three habituation trials. Once infants met this habituation criterion, they viewed four test events. The test events combined the familiarity of the objects (familiar vs. novel) and the familiarity of the spatial relation (familiar vs. novel) within each condition (e.g., infants in the simple condition viewed simple test objects and those in the complex condition viewed complex objects). Specifically, these test events were (a) Familiar objects in a familiar relation (an event seen during habituation), (b) familiar objects in a novel relation (an object pair seen during habituation in a containment relation), (c) novel objects in a familiar relation (an object pair not seen during habituation in a support relation, and (d) novel objects in a novel relation (an object pair not seen during habituation in a containment relation). The presentation order of the four test events was counterbalanced across participants. If infants were able to form a category of the support relation, they were expected to look significantly longer at the test events with a novel relation than at test events with a familiar relation, 
both when the objects were familiar and when the objects were novel.

To determine the inter-observer reliability, 7 infants $(35 \%)$ were randomly chosen from the sample and a second observer who was blind to the condition and the stimulus seen by the infants recorded the looking times offline, using the same software (Habit X 1.0), while looking at the recorded video of each infant's experiment session. This resulted in 3 infants in the simple condition and 4 infants in the complex condition. The correlation between the online and offline looking times ranged from .94 to 1.00 with the average of .98 .

\section{Results}

\section{Habituation phase}

We first compared infants' average looking time during the first three trials of habituation with their looking time to the familiar test trial (i.e., one of the habituation events presented in the test phase) to ensure that the infants had indeed habituated to the events rather than reaching the habituation criterion as an artifact. If infants truly habituated to the events, they were expected to demonstrate a significant decrease in looking to the events presented during habituation. Infants' looking times were examined in a 2 (trial: average of the first three habituation trials vs. the familiar test trial) $\times 2$ (condition: simple vs. complex) mixed-model analysis of variance (ANOVA). The analysis yielded only a significant main effect for trial, $F$ $(1,18)=227.25, p<.001, \eta_{\mathrm{p}}{ }^{2}=.93$, demonstrating that infants looked significantly less at the familiar test trial $(M=5.21 \mathrm{~s}, S D=$ $2.70 \mathrm{~s})$ than the average of their looking time to the first three habituation trials $(M=21.72 \mathrm{~s}, S D$ $=4.86 \mathrm{~s})$. That is, infants provided evidence that they indeed habituated to the events presented during the habituation phase.

Next, we examined whether the number of habituation trials differed across conditions. Possibly, infants who viewed perceptually simple objects might have taken fewer trials to reach the habituation criterion than infants viewing relatively complex objects. However, a one-way ANOVA with condition (simple vs. complex) as the between-subjects factor yielded no significant effect of condition, $F(1,18)=$ $1.18, p=.29$. Infants viewed an equivalent number of trials to meet the habituation criterion in the simple condition $(M=7.20$ trials, $S D=$ 3.80 trials) and complex condition $(M=9.30$ trials, $S D=4.79$ trials).

\section{Test phase}

Preliminary analyses were conducted to determine whether there was a significant effect of test order or sex on infants' looking times to the test trials. A three-way mixed-model ANOVA with test order as the between-subjects factor and objects and spatial relation as the within-subjects factors revealed no significant effect or interaction of test order. Also, a 2 (sex: male vs. female) $\times 2$ (objects: familiar vs. novel) $\times 2$ (spatial relation: familiar vs. novel) mixedmodel ANOVA yielded no significant effect of sex on the infants' looking times to the test trials. Therefore, the data were collapsed across these variables.

Next, we addressed the central question of the present study. Do objects matter for 14-monthold infants' formation of a support category? If infants form a category of a support relation, they should demonstrate a significant increase in looking time when a novel spatial relation is depicted in the test events than when viewing the familiarized support relation. Also, if the features of the objects significantly impact infants' formation of a support category, then there should be a significant effect of condition on infants' looking times during the test phase.

Table 1 displays the means and standard deviations of infant looking time in each condition to each test trial. Infants' looking times to the test trials were examined in a 2 (objects: familiar vs. novel) $\times 2$ (spatial relation: familiar vs. novel) $\times 2$ (condition: simple vs. complex) mixed-model ANOVA. As seen in Table 2, the analysis yielded a significant main effect for objects, $F(1,18)=9.70, p<.01, \eta_{\mathrm{p}}{ }^{2}=.35$. 
Do Simple Objects Facilitate Infants' Formation of a Spatial Category?

Table 1

The Means and Standard Deviations of the 14-Month-old Infants' Looking Time to Each Test Event in Each Condition

\begin{tabular}{llccc}
\hline \multirow{2}{*}{ Condition } & \multirow{2}{*}{ Objects } & \multicolumn{2}{c}{ Spatial relation } & \\
\cline { 3 - 4 } & & Familiar & Novel & Average \\
\hline \multirow{3}{*}{ Simple } & Familiar & $5.25(2.82)$ & $6.56(7.25)$ & $5.90(4.08)$ \\
& Novel & $7.99(3.73)$ & $15.55(12.06)$ & $11.77(7.06)$ \\
\cline { 2 - 4 } & Average & $6.62(2.65)$ & $11.06(7.56)$ & $8.84(4.73)$ \\
\hline \multirow{3}{*}{ Complex } & Familiar & $5.17(2.72)$ & $11.11(9.32)$ & $8.14(5.53)$ \\
& Novel & $10.05(8.90)$ & $13.90(7.11)$ & $11.98(6.21)$ \\
\cline { 2 - 4 } & Average & $7.61(4.89)$ & $12.51(6.95)$ & $10.06(4.60)$ \\
\hline \multirow{3}{*}{ Total } & Familiar & $5.21(2.70)$ & $8.84(8.45)$ & $7.02(4.87)$ \\
& Novel & $9.02(6.72)$ & $14.73(9.67)$ & $11.87(6.47)$ \\
\cline { 2 - 4 } & Average & $7.12(3.86)$ & $11.78(7.11)$ & $9.45(4.58)$ \\
\hline
\end{tabular}

Table 2

Analysis of Variance of Infants' Looking Times to Test Events

\begin{tabular}{|c|c|c|c|}
\hline Source & $d f$ & $F$ & \\
\hline \multicolumn{4}{|c|}{ Between subjects } \\
\hline Condition (C) & 1 & .34 & \\
\hline Error & 18 & $(87.10)$ & \\
\hline \multicolumn{4}{|c|}{ Within subjects } \\
\hline Objects $(\mathrm{O})$ & 1 & 9.70 & $* *$ \\
\hline Spatial relation $(\mathrm{R})$ & 1 & 8.84 & $* *$ \\
\hline $\mathrm{O} \times \mathrm{R}$ & 1 & .58 & \\
\hline $\mathrm{O} \times \mathrm{C}$ & 1 & .43 & \\
\hline $\mathrm{R} \times \mathrm{C}$ & 1 & .02 & \\
\hline $\mathrm{O} \times \mathrm{R} \times \mathrm{C}$ & 1 & 2.35 & \\
\hline Error $(\mathrm{O})$ & 18 & $(48.50)$ & \\
\hline Error (R) & 18 & $(49.24)$ & \\
\hline Error $(\mathrm{O} \times \mathrm{R})$ & 18 & (37.06) & \\
\hline
\end{tabular}

Note. Values enclosed in parentheses represent mean square errors.

$* p<.05 \quad * * p<.01$

Infants looked longer at the two test trials that presented novel objects $(M=11.87 \mathrm{~s}, S D=6.47$ s) than at the two test trials that presented familiar objects $(M=7.02 \mathrm{~s}, S D=4.87 \mathrm{~s})$. That is, infants discriminated between the novel pairs of objects (first seen in the test phase) and familiar pairs of objects (previously seen in the habituation phase). 
The analysis also yielded a significant main effect for spatial relation, $F(1,18)=8.84, p$ $<.01, \eta_{\mathrm{p}}{ }^{2}=.33$. Overall, infants looked significantly longer at the two test trials that presented the novel containment spatial relation $(M=11.78 \mathrm{~s}, S D=7.11 \mathrm{~s})$ than at the two test trials that presented the familiarized support spatial relation $(M=7.12 \mathrm{~s}, S D=3.86 \mathrm{~s})$.

The analysis did not yield any other significant effects. In particular, there were no significant effects of condition, indicating that infants did not differ significantly in their looking time across the simple versus complex conditions. Rather, it appears that infants formed a category of support regardless of condition.

An examination of the results in Figure 2, however, hints at a possible effect of condition with spatial relation and objects. Although this three-way interaction among the objects, spatial relation, and condition was not significant, $F$ (1, 18) $=2.35, p=.143, \eta_{\mathrm{p}}{ }^{2}=.12$, the pattern of looking in the graph suggest that infants' looking times to the familiar versus novel relation might vary with familiarity of the test objects across the two conditions. In the simple condition and when viewing familiar objects, infants looked an equal duration at the novel versus familiar relation, $F$ $(1,9)=.32, p=.59, \eta_{\mathrm{p}}{ }^{2}=.03$, whereas in the complex condition, infants looked significantly longer at the novel relation compared to the familiar relation when the objects were familiar, $F(1,9)=5.34, p<.05, \eta_{\mathrm{p}}{ }^{2}=.37$. When the test objects were novel, however, the opposite pattern seemed to emerge. In the simple condition, infants tended to look longer at the novel than the familiarized relation when the objects were novel, $F(1,9)=4.79, p=.056, \eta_{\mathrm{p}}{ }^{2}=.35$, but failed to do so in the complex condition, $F(1,9)=1.41, p$ $=.27, \eta_{\mathrm{p}}{ }^{2}=.14$. In sum, infants in the simple condition may have demonstrated stronger generalization of the spatial relation to novel objects while in the complex condition, infants may have demonstrated stronger discrimination in the change in spatial relation between familiar

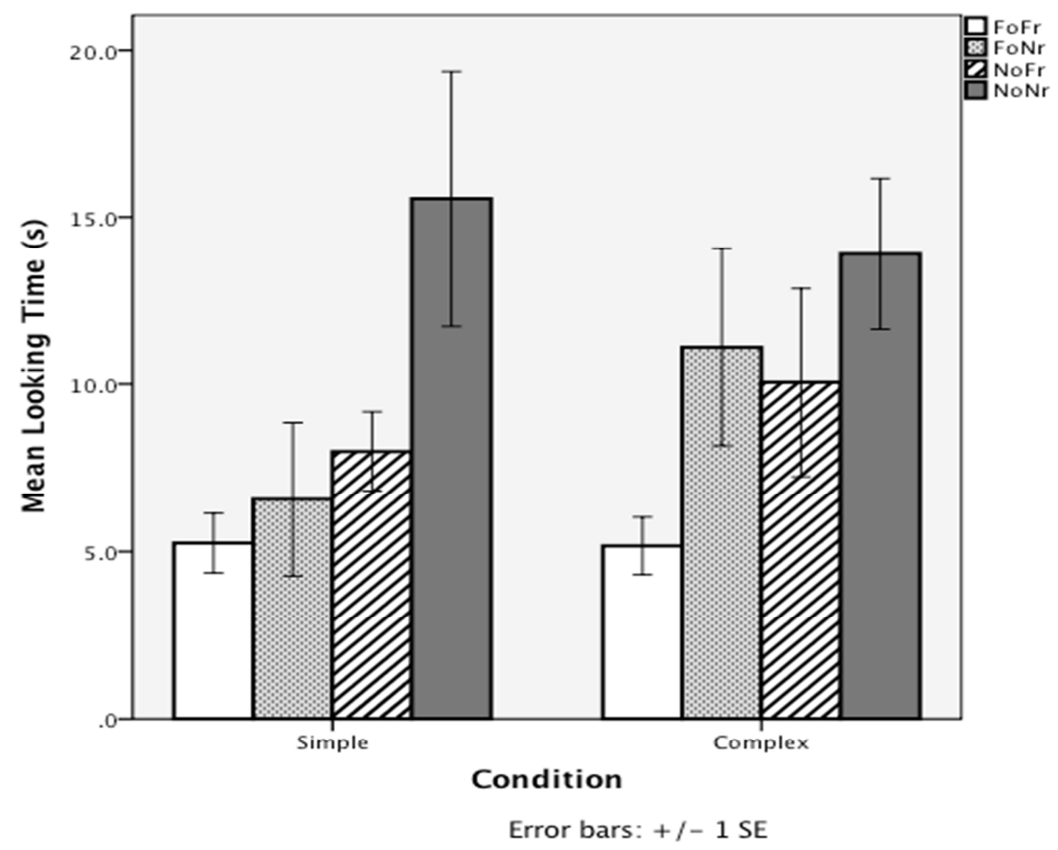

Figure 2. The looking time of 14-month-old infants to each of the four test events for infants in the simple condition (left graph) and complex condition (right graph). 
objects. However, this pattern was only somewhat suggested and remains to be examined in a second study and when more infants are included in the sample.

\section{Discussion}

The present study was designed to examine whether the perceptual simplicity of objects depicting a support relation would facilitate infants' formation of a support category. To test this possibility, we directly manipulated the perceptual simplicity of the objects through the number of features that objects in the support events depicted. We then assessed Korean 14month-old infants' formation of a support category with the perceptually simple versus complex objects. Our results did not support the hypothesis. Rather, infants of 14 months in the present task were able to form a support category regardless of the complexity of the objects' appearances. Our results additionally indicated that infants of 14 months are able to discriminate the object pairs used in the present study.

The results failed to support our initial prediction and are inconsistent with several previous findings that document an advantage of simple objects in categorization tasks (e.g., Kaminski, Sloutsky, \& Heckler, 2008; Son, Smith, \& Goldstone, 2008). However, there are a handful of research findings in agreement with the current findings. First, our result is consistent with a finding by Son, Smith, and Goldstone (2011) that perceptually simple and rich objects are equally effective at promoting children's generalization of a relation at tasks where children matched relations to samples that varied on every trial. Notably, the same researchers found that simple objects are more effective than complex ones when children matched relations to a fixed sample of a relation. In our study, infants viewed multiple exemplars of a support relation, and did not need to use and remember only one example. Thus, these findings together raise the possibility that the benefit of simplicity does not exist to the same degree in all learning tasks. Viewing an already abstracted representation (i.e., a simple instance) may be the most beneficial in types of tasks where children need to generalize a relation from only a single exemplar. In contrast, if the task allows children to experience multiple exemplars, as in our study, whether the objects are perceptually simple or complex may not significantly matter.

Secondly, the result is along a similar line with a recent finding that English-learning infants of 14 months successfully form a support category both when habituated to two exemplars and when habituated to six exemplars (Casasola \& Park, in press). Thus, it may be that by the age of 14 months, infants have gained the ability to form a support category regardless of the object information presented in the spatial categorization tasks. However, there is also a possibility of a developmental change. Although the perceptual simplicity does not matter for infants at 14 months, it may matter for younger infants, as found by Welling (2011) who noted that 3-year-old children learned words for dynamic relations somewhat better with perceptually rich objects than with simplified generic shapes while 4-year-old children showed high learning scores both with generic shapes and rich objects. Future research should address this developmental question.

On the contrary, it should be noted that the inconsistency between our results and previous findings reporting a benefit of simplicity might be attributed to the limitations of the current study. First, our study may have failed to yield a facilitative effect of simple objects because the objects in the complex condition were not sufficiently complex. Note that most of the objects used in the complex condition had a color pattern and only one or two additional small appendages such as wings or a tail. Thus, there may not have been a sufficiently pronounced perceptual difference between the simple and complex objects in the present task, explaining the null effect of condition. Also, recall that infants in the simple and complex conditions viewed an equivalent number of trials in reaching the habituation criterion. This result may reflect that the complex objects were in fact relatively simple. Hence, the present results 
cannot be taken as definitive evidence that infants' performance is not significantly hampered by complex objects. This effect remains a possibility until additional studies can examine the effect of presenting even more complex objects in the present task.

Secondly, it also should be noted that the statistical power in the present study was relatively low because of the small sample size. There was a suggested possibility in our data that infants' generalization of the spatial relation to novel objects might be more robust in the simple condition than in the complex condition. That is, the tendency that infants distinguished between the familiar and novel spatial relations when the objects were novel appeared stronger with simple than complex objects. An intriguing possibility is that with more infants, this effect might become significant. Alternatively, with more infants, the suggested three-way interaction among condition, objects and spatial relation could disappear.

Furthermore, we also consider a third possibility that object complexity and infant spatial categorization have a curvilinear relationship such that there is an optimal level of simplicity or complexity that may be distinct at different points in development. Because the current study compared infants' performance at only two levels of complexity, we could not test this possibility. This possibility remains to be tested in future research by developing stimuli to represent more points along the complexity continuum.

One might raise the question of whether there were differences in the baseline spatial abilities of infants in each condition. However, we believe that this possibility is unlikely because our data from the supplementary language survey did not reveal any significant differences in the number of words comprehended or produced between infants in the simple condition and those in the complex condition. Also, random assignment of the infants into two conditions reduces the possibility that there were any significant, systematic differences across our two randomly assigned groups of infants.

The present findings may have implications for a view that information of objects and that of relation compete for perceivers' attention. If the information of objects versus spatial relation compete for infants' attention during infants' processing of the spatial events, then the spatial relation should be best discriminated when the objects are the least interesting, namely, simple and familiar in this case. However, it was not the case in our data. Currently, with the limited sample size, infants' discrimination of spatial relation appeared very weak when the objects were familiar and simple. In contrast, when the objects were novel and simple, or when the objects were familiar and complex, infants strongly discriminated the spatial relations. When the objects were novel and complex, infants' discrimination of spatial relation again appeared relatively weak. Thus, infants may best attend to the spatial relations between the objects when the objects are neither too attentiongrabbing nor too boring and uninteresting.

Finally, the present results might be specific to our Korean sample or they might generalize to infants in other cultural groups. Because we examined the effect of object simplicity and did not examine a spatial relation that varies crosslinguistically, we believe that our results would generalize to other groups of infants. Indeed, we are currently testing infants in the US on the same task.

In conclusion, the present study demonstrates a novel finding that infants' formation of a categorical representation of a small-scale support relation is not influenced by the simplicity of object appearance. Although the results do not allow us to draw a firm conclusion about the relationship between the object complexity and infant spatial categorization, they nonetheless open a door to the future research exploring the role of objects in infants' relational learning and the most ideal learning condition.

Acknowledgements: This research was supported by NSF PECASE Award BCS0349183 and NSF BCS Award BCS-0721238 awarded to Dr. Marianella Casasola. We thank the directors and teachers at the child daycares who helped us find eligible infants. We also 
thank the parents and infants who generously gave their time to participate in this study.

\section{References}

Amaya, M., Uttal, D. H., O’Doherty, K. D., Liu, L. L., \& DeLoache, J. S. (2008). Two-Digit Subtraction: Examining the link between concrete and abstract representations of knowledge. Manuscript under revision.

Behl-Chadha, G., \& Eimas, P. D. (1995). Infant categorization of left-right spatial relations. British Journal of Developmental Psychology, 13, 69-79.

Casasola, M. (2005). When less is more: How infants learn to form an abstract categorical representation of support. Child Development, 76, 279-290.

Casasola, M. (2008). The development of infants' spatial categories. Current Directions in Psychological Science, 17, 21-25.

Casasola, M., \& Cohen, L. B. (2002). Infant categorization of containment, support, and tight-fit spatial relationships. Developmental Science, 5, 247-264.

Casasola, M., Cohen, L. B., \& Chiarello, E. (2003). Six-month-old infants' categorization of containment spatial relations. Child Development, 74, 679-693.

Casasola, M., \& Park, Y. (in press). Developmental changes in infant spatial categorization: When more is best and when less is enough. Child Development.

Choi, S. (2006). Influence of language-specific input on spatial cognition: Categories of containment. First Language, 26, 207-232.

Choi, S., \& Bowerman, M. (1991). Learning to express motion events in English and Korean: The influence of language-specific lexicalization patterns. Cognition, 41, 83-121.

Cohen, L. B., Atkinson, D. J., \& Chaput, H. H. (2004). Habit X: A new program for testing infant perception and cognition [Computer software]. Austin, the University of Texas.

Gava, L., Valenza, E., \& Turati, C. (2009). Newborns' perception of left-right spatial relations. Child Development, 80, 1797-1810.
Kaminski, J. A., \& Sloutsky, V. M. (2010) Concreteness and relational matching in preschoolers. In S. Ohlsson \& R. Catrambone (Eds.), Proceedings of the XXXII Annual Conference of the Cognitive Science Society (pp. 335-340). Mahwah, NJ: Erlbaum.

Kaminski, J. A., Sloutsky, V. M., \& Heckler, A. F. (2006). Do children need concrete instantiations to learn an abstract concept? In R. Sun and N. Miyake (Eds.). Proceedings of the XXVIII Annual Conference of the Cognitive Science Society (pp. 411-416).

Kaminski, J. A., Sloutsky, V. M., \& Heckler, A. F. (2008). The advantage of abstract examples in learning math. Science, 320, 454-455.

Maguire, M., Hirsh-Pasek, Golinkoff, R. (2008). Focusing on the relation: Fewer exemplars facilitate children's initial verb learning and extension. Developmental Science, 11, 628634.

McDonough, L., Choi, S., \& Mandler, J. (2003). Understanding spatial relations: Flexible infants, lexical adults. Cognitive Psychology, 46, 229-259.

Paik, J. H., \& Mix, K. (2006). Preschoolers' use of surface similarity in object comparisons: Taking context into account. Journal of Experimental Child Psychology, 95, 194-214.

Park, Y., \& Casasola, M. (in preparation). Do objects matter for infants' formation of a spatial category?

Petersen, L. A., \& McNeil, N. M. (2008). Using perceptually rich objects to help children represent number: Established knowledge counts. In V. Sloutsky, B. Love, \& K. McRae (Eds.), Proceedings of the Thirtieth Annual Conference of the Cognitive Science Society (p. 1567- 1572). Mahwah, NJ: Erlbaum.

Quinn, P. C. (1994). The categorization of above and below spatial relations by young infants. Child Development, 65, 58-69.

Quinn, P. C., Adams, A., Kennedy, E., Shettler, L., \& Wasnik, A. (2003). Development of an abstract category representation for the spatial relation between in 6- to 10-month-old infants. Developmental Psychology, 39, 151-163.

Quinn, P. C., Cummins, M., Kase, J., Martin, E., 
\& Weissman, S. (1996). Development of categorical representations for above and below spatial relations in 3- to 7-month-old infants. Developmental Psychology, 32, 942950.

Quinn, P. C., Polly, J., Furer, M., Dobson, V., \& Narter, D. (2002). Young infants' performance in the object-variation version of the abovebelow categorization task: A result of perceptual distraction or conceptual limitation? Infancy, 3, 323-347.

Rattermann, M. J., Gentner, D., \& DeLoache, J. (1990). The effects of familiar labels on young children's performance in an analogical mapping task. In Proceedings of the $12^{\text {th }}$ annual conference of the cognitive science society (pp.22-29). Hillsdale, NJ: Lawrence Erlbaum.

Son, J. Y., Smith, L., \& Goldstone, R. (2008). Simplicity and generalization: Short-cutting abstraction in children's object categorizations. Cognition, 108, 626-638.
Son, J. Y., Smith, L., \& Goldstone, R. (2011) Connecting instances to promote children's relational reasoning. Journal of Experimental Child Psychology, 108, 260-277.

Uttal, D. H., Scudder, K. V., \& DeLoache, J. S. (1997). Manipulatives as symbols: A new perspective on the use of concrete objects to teach mathematics. Journal of Applied Developmental Psychology, 18, 37-54.

Welling, C. (2011). Is it daxing? The effect of learning formats on preschoolers' acquisition of novel dynamic relations. Honors research thesis. Ohio State University.

Received July 27, 2012

Revision Received August 15, 2012

Accepted August 17, 2012 